\title{
Estudo Comparativo de Abordagens Referentes ao Desenvolvimento do Pensamento Computacional
}

\author{
Daniel Cândido ${ }^{1}$, Gabriele Pessoa ${ }^{1}$, Bruna Vasconcelos $^{1}$, Klebson Silva ${ }^{1}$, \\ Renato Oliveira $^{1}$, Maurício Taumaturgo ${ }^{2}$, Taciana Pontual Falcão ${ }^{1}$ \\ ${ }^{1}$ DEINFO - Universidade Federal Rural de Pernambuco (UFRPE) - \\ Rua Dom Manoel de Medeiros, s/n - Dois Irmãos, Recife - PE - Brasil \\ ${ }^{2}$ Centro de Estudos e Sistemas Avançados do Recife (CESAR), Recife - PE - Brasil \\ \{candidosdaniel, gabriele.pessoa, brunapvasconcelos, klebson.a7d, \\ b.renatodeoliveira\}@gmail.com, mto@cesar.org.br, \\ taciana.pontual@ufrpe.br
}

\begin{abstract}
The aim of this paper is to describe a comparative study between two approaches for the development of computational thinking in basic education, specifically analyzing the concept of functions in programming. The method adopted was the application of the digital game Lightbot and a body simulation activity, in different order, combined with pre and post tests, aiming to investigate learning differences related to the order of application of such teaching methods. Results varied according to the classes, with significant improvement in students' comprehension, pointing to relevant directions for educators seeking alternative methods for teaching programming.
\end{abstract}

Resumo. Este artigo tem por objetivo descrever um estudo comparativo entre duas abordagens para o desenvolvimento do pensamento computacional na educação básica, analisando especificamente o conceito de funções na programação. $O$ método adotado foi a aplicação do jogo digital Lightbot e de uma atividade de simulação corporal, em ordens diferentes, combinados a pré e pós-testes, visando investigar as diferenças de aprendizagem relacionadas à ordem de aplicação desses métodos de ensino. Os resultados variaram de acordo com as turmas, com uma melhora significativa no entendimento por parte dos educandos, apontando direções relevantes para educadores em busca de alternativas inovadoras para o ensino de programação.

\section{Introdução}

O desenvolvimento tecnológico da computação no século XXI introduziu à sociedade um paradigma moderno, onde os cidadãos têm nas mãos o poder de criar e reproduzir tecnologia. Com isso, a lista de habilidades necessárias para o exercício pleno da cidadania foi acrescida com o Pensamento Computacional (PC), que pode ser definido como a apropriação de técnicas e habilidades da computação para resolução de problemas [Wing 2006] e pontuado como, talvez, a mais importante e menos compreendida das habilidades necessárias ao cidadão contemporâneo [Blikstein 2008]. No Brasil, entretanto, tem-se reservado aos cursos superiores o uso da computação para a produção de ciência e tecnologia, enquanto que o acesso social de massa limita-se à 
utilização do que é desenvolvido. Ao analisar o cenário da integração do PC ao contexto escolar, destaca-se a necessidade de desenvolver e aprimorar novas metodologias para o ensino de programação.

A pesquisa aqui apresentada foi realizada no Pernambucoders, um projeto extracurricular que integra o ensino de programação na educação básica pública, no formato de clubes de programação no contraturno, promovendo a produção de artefatos digitais que solucionem demandas educacionais e sociais da realidade dos estudantes, e fortalecendo o processo de ensino-aprendizagem por meio da integração de disciplinas. Várias abordagens são utilizadas nos clubes, tais como aulas expositivas, ambientes de programação visual, atividades de computação desplugada e jogos. Entretanto, por se tratar de uma iniciativa recente, os educadores ainda estão experimentando práticas de ensino e buscando as melhores formas de combinar abordagens.

Visando contribuir para essa investigação, este artigo apresenta um estudo comparativo em clubes de programação de três escolas de ensino fundamental e médio, envolvendo a aplicação de duas metodologias lúdicas para o ensino de conceitos de programação e desenvolvimento do PC. Foram usados a simulação corporal e um jogo digital para introduzir os conceitos de funções e procedimentos, alternando-se a ordem de aplicação para investigar se haveria diferença na compreensão dos alunos. Este trabalho está organizado da seguinte forma: a Seção 2 apresenta um levantamento acerca de atividades desplugadas e jogos digitais para o ensino de programação; a Seção 3 apresenta os procedimentos metodológicos utilizados e as atividades desenvolvidas; a Seção 4 trata dos resultados obtidos e as referentes discussões; e a Seção 5 expõe as considerações finais, com as conclusões e planejamentos para trabalhos futuros.

\section{Trabalhos Relacionados}

Metodologias lúdicas, que abordam conceitos básicos de programação para o desenvolvimento do PC, são amplamente encontradas na literatura [Zanetti et al. 2016]. No Brasil, as práticas mais utilizadas para este fim têm sido as linguagens de programação visual, jogos digitais e computação desplugada. Estas práticas apresentam em comum a ludicidade, em contraste a ambientes tradicionais de programação.

A utilização de jogos digitais para o desenvolvimento do PC tem trazido resultados positivos como a diminuição no índice de evasão, aumento na motivação e melhora no desempenho dos alunos em disciplinas de introdução a programação [Silva et al. 2014]. Trabalhos recentes [Ferreira et al. 2015a, Gomes et al. 2016, Raabe et al. 2015] evidenciam as vantagens de se utilizar ferramentas como Scratch, Code.Org, Lightbot e The Foos ${ }^{1}$ no ensino-aprendizagem de conteúdos de programação para crianças e adolescentes. Para a investigação aqui apresentada, foi escolhido o jogo Lightbot, levando em consideração a opinião de outros educadores dos clubes de programação que testaram diversas ferramentas, chegando ao senso comum de que o ambiente em questão era o melhor para o ensino de funções. Esse tipo de atividade acaba envolvendo o indivíduo nas características cognitiva, emocional e relacional no processo de ensino-aprendizagem, possibilitando criatividade e desenvolvimento de competências para solução de problemas [Borges 2005].

1 https://scratch.mit.edu/, https://code.org/, https://lightbot.com/flash.html, http://thefoos.com/ 
Outro método lúdico e bastante popular utilizado para auxiliar a compreensão de conceitos da computação e o desenvolvimento do PC, no entanto sem a dependência do computador, baseia-se no livro "Computer Science Unplugged" [Bell et al. 2009] (Computação Desplugada). Atividades desplugadas facilitam o entendimento de assuntos básicos, possibilitando também a ligação a outros assuntos mais complexos [Ferreira et al. 2015b]. As atividades de computação desplugada envolvem materiais concretos e brincadeiras, mas poucos trabalhos adotam como técnica a simulação corporal aqui utilizada. Esta técnica permite deslocar o aluno do laboratório tradicional para um ambiente aberto e modular, como pátio ou auditório, e despertar o lado artístico e criativo [Vieira et al. 2013]. Assim, tira o estudante da zona de conforto e o coloca em uma posição ativa, trabalhando com emoções e principalmente com o improviso, que desperta a capacidade de lidar com situações do mundo real ou imaginário, envolvendo movimentos corporais.

\section{Metodologia}

A presente pesquisa teve como objetivo investigar se a ordem de aplicação de atividades de natureza distintas tem influência na compreensão sobre funções, no contexto das aulas de um clube de programação. $O$ tema funções em programação foi escolhido por conta de dificuldades dos educandos, percebidas de forma empírica pelos educadores, autores deste artigo, com turmas anteriores dos clubes. Para investigar possibilidades didáticas para melhorar a aprendizagem deste conteúdo, foram escolhidas atividades alinhadas a duas abordagens distintas: simulação corporal (atividade desplugada) e jogo digital (atividade no computador). Essas abordagens já são aplicadas nos clubes em diferentes conteúdos, mas não está claro, na prática dos educadores, se existe e qual seria a ordem ideal de aplicação para promover uma melhor compreensão.

A pesquisa foi aplicada em 3 turmas (uma com cerca de 20 estudantes, e duas com aproximadamente 30 estudantes) de clubes de programação de 3 escolas públicas. Os educandos envolvidos nos clubes de programação têm sua participação autorizada pelos responsáveis. Além disso, foi firmado nesta pesquisa o compromisso de garantir o anonimato dos estudantes. Os educandos avaliados já tinham conhecimento dos conceitos de algoritmos, variáveis, estruturas condicionais e de repetição, porém não tinham nenhum conhecimento prévio sobre o conteúdo de funções.

\subsection{Procedimento}

A pesquisa foi realizada nos meses de abril e maio de 2017, pelos educadores responsáveis por cada turma (autores deste artigo). As atividades aconteceram durante as aulas dos clubes de programação, em dois encontros sequenciais de 3 horas de duração cada, respeitando o cronograma dos conteúdos previstos. $\mathrm{O}$ primeiro encontro consistiu na aplicação de um pré-teste e um tipo de atividade, e no segundo foi realizada a atividade da outra abordagem, seguida de um pós-teste. Considerando a diferença na quantidade de alunos por turma em cada escola, para efeitos de comparação foi adotada a mesma ordem de atividades nas Escolas 1 e 2, e na Escola 3 a ordem inversa. Assim, nas Escolas 1 e 2, as atividades foram aplicadas na seguinte ordem: (i) Pré-teste; (ii) Simulação corporal; (iii) Jogo Lightbot; (iv) Pós-teste. Na Escola 3, houve a inversão na ordem das etapas (ii) e (iii). Apesar do planejamento para manter os grupos que realizaram as duas abordagens com tamanhos próximos, um desafio enfrentado foi a 
VI Congresso Brasileiro de Informática na Educação (CBIE 2017)

Anais do XXIII Workshop de Informática na Escola (WIE 2017)

variação entre os estudantes presentes em cada encontro, como mostra a Tabela 1. Dado o objetivo da pesquisa, apenas os dados dos estudantes presentes nos dois encontros foram analisados. Assim, os grupos comparados contaram com 18 e 13 alunos.

Tabela 1. Quantidade de estudantes participantes em cada encontro

\begin{tabular}{|c|cccc|}
\cline { 2 - 5 } \multicolumn{1}{c|}{} & Encontro 1 & Encontro 2 & Participantes & Analisados \\
\hline Escola 1 & 10 & 11 & 11 & 10 \\
Escola 2 & 30 & 21 & 31 & 8 \\
Escola 3 & 20 & 19 & 25 & 13 \\
\hline
\end{tabular}

\subsection{Atividades Realizadas}

As atividades propostas por este trabalho tiveram como finalidade a abordagem lúdica do conteúdo de funções em programação. As duas atividades abordam características referentes aos conceitos teóricos e práticos da utilização de funções como recurso importante na programação.

\subsubsection{Atividade com o Jogo Lightbot}

O Lightbot é um jogo educacional que aborda conceitos de programação através de blocos de instrução. O objetivo do jogo é comandar um robô, para que ele se movimente em um cenário dividido em plataformas cúbicas e acenda todas as plataformas indicadas em azul, como mostra a Figura 1. O jogador tem a sua disposição blocos de comandos representados por símbolos, na parte inferior esquerda da interface. No primeiro nível do jogo, os comandos disponíveis, são: andar para frente, acender a plataforma, virar à esquerda, virar à direita e pular para frente. Do lado direito, é disponibilizado um espaço $(M A I N)$ para que o jogador possa arrastar os blocos disponíveis a fim de desenvolver um algoritmo para solucionar os problemas apresentados a cada fase.
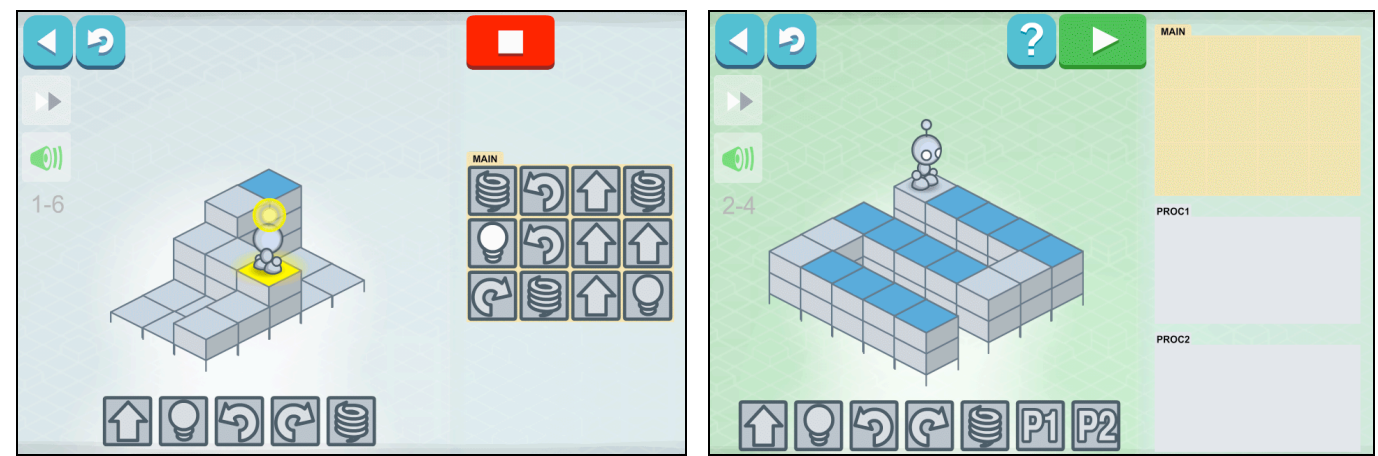

Figura 1. Telas do jogo Lightbot

O principal desafio do jogo é encontrado na limitação dos espaços para inserção de comandos, fazendo com que o jogador precise otimizar o algoritmo planejado de forma a atingir o objetivo com o mínimo de instruções possível. No segundo nível, é verificada a motivação principal da escolha deste jogo para este trabalho, pois são apresentados novos recursos e comandos para a utilização do jogador (Figura 1, à direita). Dois novos blocos de comandos são disponibilizados: P1 e P2. Estes dois blocos são referências de execução aos dois novos espaços para inserção de código: PROC1 e PROC2. Deste modo, as novas fases exigem que o jogador utilize estes novos espaços e seus respectivos blocos de referência a fim de conseguir êxito, criando 
instruções de forma personalizada, a partir do seu entendimento sobre o problema. Nestas ações, o jogador trabalha intuitiva e diretamente com o conceito de modularização de problemas, reuso de código e funções na programação. A atividade com o Lightbot foi aplicada da seguinte forma:

I. Disponibilizar o jogo nos computadores dos educandos;

II. Informar aos educandos a possibilidade de questionar os educadores com relação a qualquer dúvida referente à jogabilidade. Porém, não podendo ser respondidas questões com relação ao conteúdo ou resolução dos problemas apresentados no jogo;

III. Solicitar aos educandos que joguem o jogo até o nível 2 - referente aos recursos de P1 e P2 que representam funções. Após isto, liberá-los para jogar livremente.

\subsubsection{Atividade de Simulação Corporal}

Uma atividade de simulação corporal consiste na representação de conceitos com movimentos, posicionamentos e ações realizadas pelos participantes com os próprios corpos. Como tema para a simulação proposta foram escolhidos 4 jogos tradicionais da história dos jogos eletrônicos para a representação a ser realizada pelos educandos, sendo eles: Pac-Man, Snake II, Space Invaders e Pong ${ }^{2}$. Além da possibilidade de serem utilizados como ferramentas de ensino-aprendizagem [Antunes 1998], esses jogos também possuem uma grande referência histórica por se tratarem de jogos conhecidos com características de fácil entendimento e de simples jogabilidade. Para realização desta atividade, foram adotados os seguintes passos:

I. Preparar um ambiente espaçoso para os ensaios e a realização das simulações;

II. Apresentar os quatro jogos, em forma de vídeos, e disponibilizar o endereço online dos jogos para que os estudantes possam jogá-los e conhecê-los melhor;

III. Dividir os estudantes em equipes de 4 a 6 integrantes, de maneira a equilibrar a quantidade de educandos por grupo de acordo com o jogo escolhido (caso a turma não tenha uma quantidade suficiente, recomendase utilizar apenas 3 jogos);

IV. Atribuir, de maneira sorteada, os jogos a cada equipe, sem repetições;

V. Determinar um tempo de 40 minutos para o desenvolvimento da simulação corporal dos jogos;

VI. Apresentação das equipes, sendo 2 minutos para a apresentação de como foi construída a atividade e 6 minutos para a simulação do jogo. $\mathrm{Na}$ simulação, os educadores deverão interferir para que fique claro para todos as funções que foram utilizadas.

Nesta atividade, a simulação corporal foi utilizada para representar e abstrair os conceitos de funções, como: modularização de problemas, divisão de encargos para tarefas específicas e interação entre módulos do sistema. Estes conceitos são trabalhados pelos educandos à medida que são definidas e executadas suas próprias funções e, também, quando estas funções individuais interagem causando uma reação programada.

2 http://originalpacman.com, http://helpfulsheep.com/snake, http://www.pacxon4u.com/space-invaders, http://www.ponggame.org 
VI Congresso Brasileiro de Informática na Educação (CBIE 2017)

Anais do XXIII Workshop de Informática na Escola (WIE 2017)

Além disso, os educandos devem responder de forma clara e objetiva, através dos seus movimentos, "Qual a sua função no jogo?", "Quais movimentos cada um deve executar?" e "Quais espaços devem ocupar?".

\subsection{Avaliação}

O método de avaliação escolhido foi por meio de pré e pós-teste [Dimitrov e Rumrill 2003] para analisar o nível de acerto dos educandos antes e depois das atividades realizadas. As quatro perguntas que constituem os pré e pós-testes foram elaboradas para que indiretamente direcionassem os alunos a responderem pensando em situações do cotidiano ou já vividas por eles, já que até então, não sabiam do que se tratava uma função a não ser pelo significado da palavra. O pré e pós-teste foram muito similares, apenas com modificações nos tempos verbais. Os testes foram aplicados em papel na Escola 1, por conta da indisponibilidade de acesso à internet no local, e em formulário online nas Escolas 2 e 3. Foram realizadas análises quantitativa e qualitativa acerca das 4 questões referentes aos questionamentos sobre funções. Para cada questão, foram definidas expectativas de respostas como parâmetros de acerto (Tabela 2).

Tabela 2. Questões dos testes

\begin{tabular}{|c|c|c|c|}
\hline ID & Questão & Tipo & Expectativa de Resposta \\
\hline Q1 & $\begin{array}{l}\text { O que você acha que é uma } \\
\text { Função? Você teria algum } \\
\text { exemplo do seu cotidiano? } \\
\text { Qual? }\end{array}$ & Aberta & $\begin{array}{l}\text { Tarefas individuais como: sou estudante e minha } \\
\text { função é estudar, ir para a escola. Em casa, } \\
\text { minha função é lavar os pratos. A função do } \\
\text { professor é ensinar. }\end{array}$ \\
\hline Q2 & $\begin{array}{l}\text { Marque a(s) opção(ões) que } \\
\text { você acha que pode }(\mathrm{m}) \text { definir } \\
\text { uma Função: }\end{array}$ & $\begin{array}{l}\text { Múltipla } \\
\text { escolha }\end{array}$ & $\begin{array}{l}\text { - Algo que pode ser reaproveitado para várias } \\
\text { situações; } \\
\text { - Algo que representa uma tarefa específica. }\end{array}$ \\
\hline Q3 & $\begin{array}{l}\text { De acordo com o texto, } \\
\text { identifique o que poderia ser } \\
\text { uma Função e a descreva em } \\
\text { linguagem natural: }\end{array}$ & Aberta & $\begin{array}{l}\text { Almoçar; Atender o carteiro; Entregar as } \\
\text { correspondências; Empurrar o carro; Dar partida } \\
\text { na ignição; Lavar os pratos; Corrigir as provas. E } \\
\text { uma descrição em Linguagem Natural de uma } \\
\text { dessas funções. }\end{array}$ \\
\hline Q4 & $\begin{array}{l}\text { O que você imagina que seja } \\
\text { Função na programação e para } \\
\text { que serve? }\end{array}$ & Aberta & $\begin{array}{l}\text { Um trecho do código/programa que pode ser } \\
\text { reaproveitado em diversos momentos, que } \\
\text { representa atividades específicas, que divide o } \\
\text { problema em várias partes. }\end{array}$ \\
\hline
\end{tabular}

$\mathrm{Na}$ pergunta de múltipla escolha, as demais alternativas, definidas como incorretas, foram: Algo que causa ou gera uma repetição; Algo que representa uma sequência de passos; Algo utilizado para armazenar valores.

\section{Resultados Obtidos e Discussões}

A fim de deixar mais clara a comparação entre as duas abordagens utilizadas, considerase nesta seção como Grupo A as escolas 1 e 2, que vivenciaram a atividade de simulação corporal seguida da atividade com o jogo Lightbot; e Grupo B a escola 3, que vivenciou as atividades na ordem inversa.

De forma a analisar as questões do tipo abertas em relação às expectativas de respostas (Tabela 2) pré-definidas, as respostas dos estudantes foram classificadas em: Consistentes, Pouco Consistentes e Inconsistentes; sendo as consistentes, respostas 
equivalentes; pouco consistentes, respostas aproximadas; e inconsistentes, respostas fora do contexto. Na questão de múltipla escolha, os três níveis foram mantidos a fim de padronizar a análise dos dados. Para isso, nessa questão a resposta foi considerada Consistente se o educando assinalou as duas opções consideradas corretas (Tabela 2); Pouco Consistente se assinalou apenas uma das corretas; e Inconsistente se não assinalou nenhuma das duas. Os dados foram analisados por todos os educadores pesquisadores, a fim de obter o maior consenso possível nas avaliações.

Para a comparação do desempenho dos estudantes dos dois grupos nos pré e pós-testes, foram analisadas as transições entre as categorias de resposta definidas. Por exemplo, aumentar 1 nível corresponde ao fato do educando responder de forma Inconsistente no pré-teste e de forma Pouco Consistente no pós-teste, e uma melhora de 2 níveis corresponde à evolução de Inconsistente para Consistente.

Conforme pode ser visto na Figura 2, no Grupo A 50\% dos estudantes permaneceram no mesmo nível, seguida de uma melhora (de 1 e de 2 níveis) de $34 \%$ na Questão 1. Na Questão 2, que apresenta o maior número de conhecimentos constantes, não foi observado nenhum nível de melhora. Já na Questão 3, houve dois empates: um de 33\% entre os estudantes que apresentaram melhora de 1 nível e os que não apresentaram avanço ou declínio; e um empate de $17 \%$ entre os alunos que apresentaram melhora de 2 níveis e os que pioraram 1 nível. Por fim, na Questão 4, mais da metade dos estudantes obtiveram avanço de 1 ou 2 níveis, 39\% permaneceram constantes e apenas $6 \%$ apresentaram piora de 1 nível.

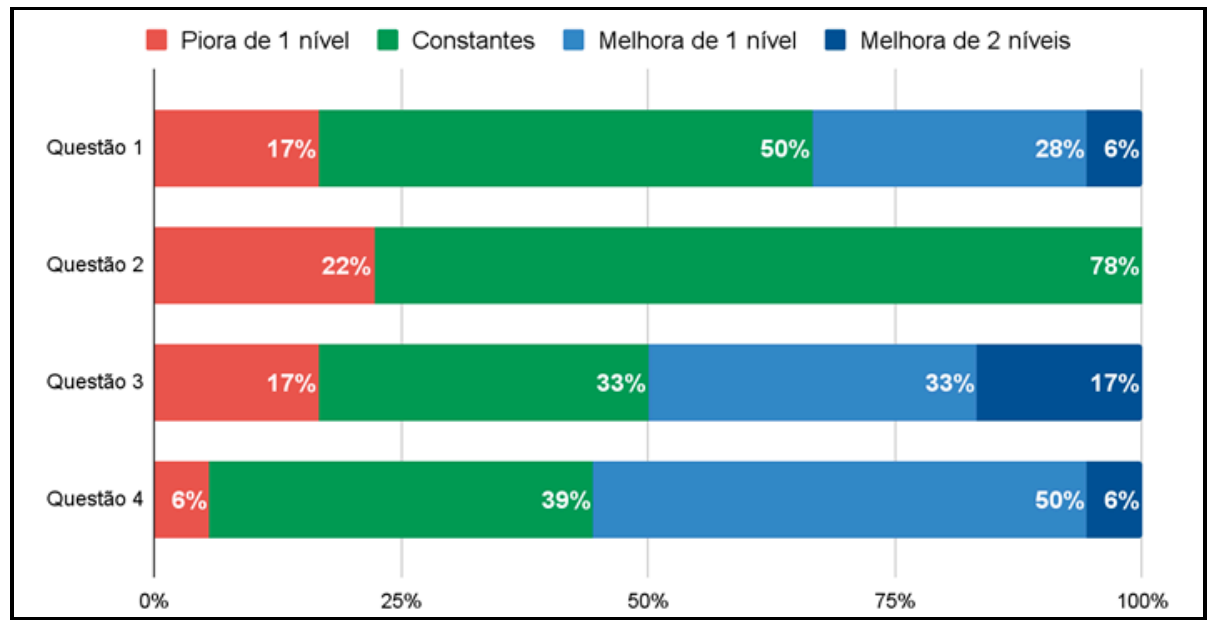

Figura 2. Análise dos dados - Grupo A (escolas 1 e 2)

A análise no Grupo B se apresentou de forma diferente. Conforme apresentado na Figura 3, na Questão 1, foi observado que 62\% dos estudantes conseguiram melhoras de 1 nível, $8 \%$ apresentaram melhoras de 2 níveis e $23 \%$ permaneceram no atual nível de aprendizado. A evolução não persistiu na Questão 2, onde o número de aprendizado constante sobe para $85 \%$ e o de melhoria de 1 nível cai para $15 \%$. A constante permanece maior na Questão 3 com $62 \%$ e $54 \%$ na Questão 4 contra $31 \%$ dos estudantes que evoluíram 1 nível na Questão 3 e 38\% da mesma evolução na Questão 4. Entretanto, no Grupo B só houve incidências de piora de 1 nível nas Questões 1 e 3, por $8 \%$ dos alunos, um número muito pequeno. 
VI Congresso Brasileiro de Informática na Educação (CBIE 2017)

Anais do XXIII Workshop de Informática na Escola (WIE 2017)

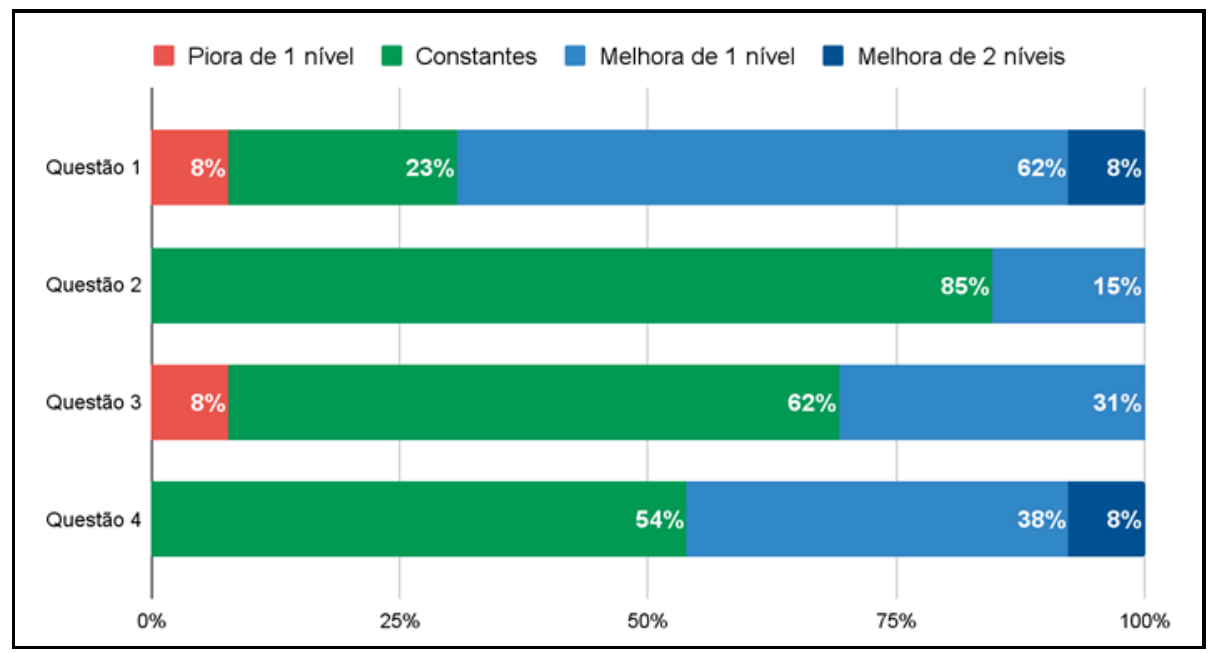

Figura 3. Análise dos dados - Grupo B (escola 3)

Além disso, foi observado em todas as questões que grande parte dos educandos que permaneceram constantes já apresentou inicialmente um bom conhecimento empírico, ou seja, respondeu de forma Consistente ou Pouco Consistente já no pré-teste e isso permaneceu no pós-teste. Na questão 2 no Grupo A, e nas questões 1, 2 e 3 no Grupo B, todos os estudantes que permaneceram com desempenho constante já tinham respondido às questões de forma Pouco Consistente ou Consistente no pré-teste e mantiveram seu nível de acerto no pós-teste.

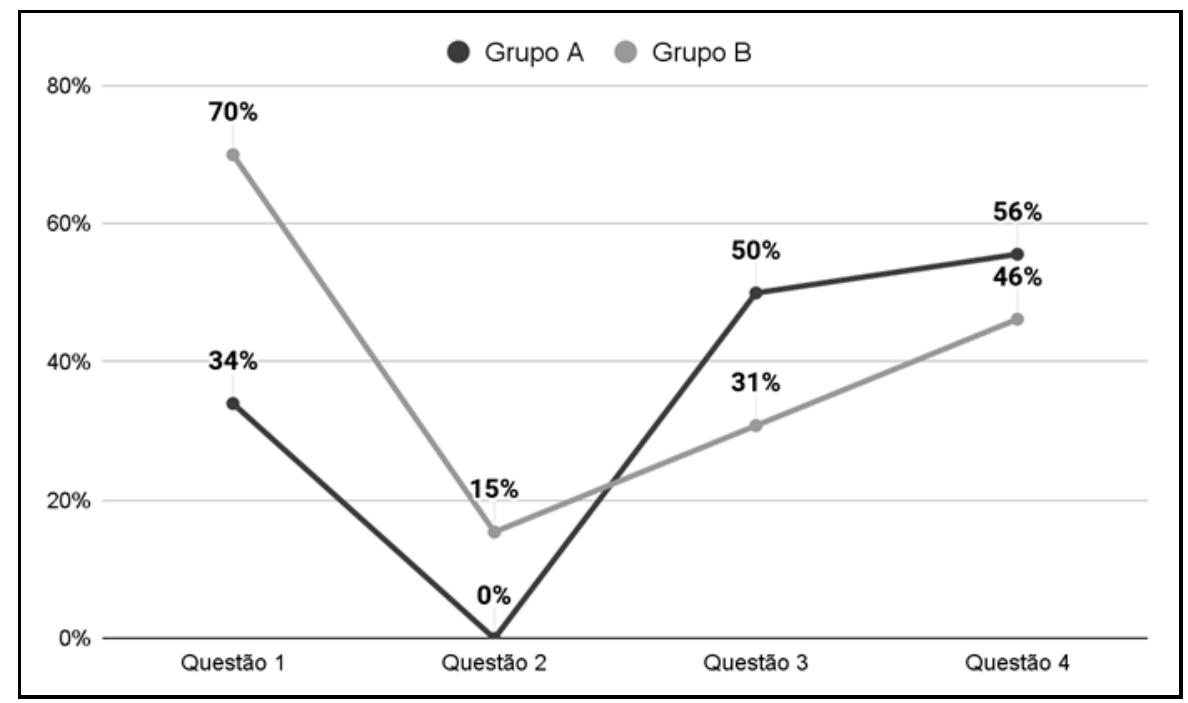

Figura 4. Comparação dos percentuais de melhora (Grupo A x Grupo B)

$\mathrm{Na}$ Figura 4, é possível observar os percentuais de melhora no desempenho dos educandos nos Grupos A e B. No grupo A, cuja ordem de aplicação das atividades foi (i) simulação corporal e (ii) Lightbot, o índice de melhoria foi melhor nas Questões $3 \mathrm{e}$ 4. Em contrapartida, no Grupo B cuja ordem foi (i) Lightbot e (ii) simulação corporal, houve um maior índice de evolução nas Questões 1 e 2. Através das informações contidas na Figura 4, foi contabilizado uma média de melhoria de 35\% no Grupo A e $40,5 \%$ no Grupo B, indicando que a ordem aplicada no Grupo B apresentou resultados mais satisfatórios do ponto de vista qualitativo. 
É notório que na Questão 1, no Grupo B, houve o mais alto índice de melhora de toda a pesquisa (70\%). Uma conclusão possível é a de que a última atividade e, portanto, mais recente para os educandos na resposta ao pós-teste, aplicada neste grupo foi a de simulação corporal, que trabalha abstratamente com o conceito de funções. Assim, o estabelecimento da relação de funções com situações do cotidiano pôde ser maior, visto que a Questão 1 trata exatamente de um questionamento sobre funções no dia-a-dia do educando. Como exemplo, pode ser apresentada a seguinte resposta no préteste, considerada Inconsistente, de um educando do Grupo B: "É desempenhar uma ação". Já no pós-teste, o mesmo educando respondeu de forma Consistente: "É o ato de executar ações para realizar um determinado problema ou tarefa. Um exemplo pode ser a escova de dentes que foi criada com uma única função que é a de escovar os dentes".

Outra observação é a de que o Grupo A apresentou o seu maior índice de melhora na Questão $4(56 \%)$. Isso também pode ser relacionado com a aplicação ao final do experimento da atividade com o jogo Lightbot, que insere o estudante em um ambiente digital e de experiências mais técnicas, ou seja, com práticas mais próximas do conceito de funções na programação, indagado no questionamento da Questão 4. Um exemplo desse tipo de melhoria pode ser visto nesta resposta no pré-teste, considerada Inconsistente, de um educando do Grupo A: "Um site usado para fazer programação de jogos e apps". No pós-teste, o educando apresentou uma melhora de 2 níveis, com a seguinte resposta: "É uma sintonia de códigos ou comandos para realizar certa tarefa".

\section{Considerações Finais}

O presente trabalho buscou investigar os impactos na forma com que se introduz um conceito de programação (funções), utilizando uma atividade offline e uma online, analisando a ordem de aplicação. Os resultados da comparação entre a ordem de aplicação de abordagens de simulação corporal e jogos digitais para a aprendizagem de funções mostraram-se inconclusivos, porém a avaliação realizada por meio de pré e póstestes permitiu identificar um aumento eficaz de conhecimento em ambas as ordens, mesmo que em questões diferentes dos testes. Há diversas limitações que influenciam esses resultados, tais como: variação na mediação dos diferentes educadores; variação nos perfis dos grupos das três escolas; quantidade pequena de estudantes que participaram dos dois encontros; e curto tempo para realização do experimento. Assim, em trabalhos futuros, objetiva-se a aplicação destas atividades em outras turmas, em um tempo mais longo e com avaliações mais profundas. Espera-se também aplicar estas estratégias em todas as outras escolas participantes do projeto com a finalidade de encontrar contribuições para o aprimoramento de metodologias do ensino de funções com a inclusão da coleta de relatos e sugestões dos estudantes.

\section{Agradecimentos}

O projeto PERNAMBUCODERS é uma colaboração entre a Secretaria de Educação de Pernambuco (SEE) e o Núcleo de Gestão do Porto Digital (NGPD) que conta com parceria do C.E.S.A.R. - Centro de Estudos e Sistemas Avançados do Recife, UFRPE Universidade Federal Rural de Pernambuco e o Softex Recife - Centro de Excelência em Tecnologia de Software do Recife. Agradecemos o apoio de Polyana Targino e Felipe Furtado para a realização dessa pesquisa e a Danielle Gomes pela inspiração. 
VI Congresso Brasileiro de Informática na Educação (CBIE 2017)

Anais do XXIII Workshop de Informática na Escola (WIE 2017)

\section{Referências}

Antunes, C. (1998). Jogos para estimulação das múltiplas inteligências. Petrópolis, RJ: Editora Vozes.

Bell, T.; Alexander, J.; Freeman, I. e Grimley, M. (2009). Computer science unplugged: School students doing real computing without computers. The New Zealand Journal of Applied Computing and Information Technology, 13(1), 20-29.

Borges, R. M. R. e Schwarz, V. (2005). O Papel dos jogos educativos no processo de qualificação de professores de ciências. Iv Encontro Ibero-americano de Coletivos Escolares e Redes de Professores que fazem Investigação na Escola.

Blikstein, P. (2008). O pensamento computacional e a reinvenção do computador na educação.

Dimitrov, D. M. e Rumrill Jr, P. D. (2003). Pretest-posttest designs and measurement of change. Work, 20(2), 159-165.

Ferreira, M. A.; de Lima, J. S.; Santos, H. M.; Oliveira, E., e do Nascimento, J. M. F. (2015a). Computação para Ensino Médio na Modalidade Semipresencial: Uma Experiência da Disciplina de Estágio Supervisionado. In: Workshop de Informática na Educação (WIE, 2015).

Ferreira, A. C.; Melhor, A.; Barreto, J.; de Paiva, L. F. e Matos, E. (2015b). Experiência prática interdisciplinar do raciocínio computacional em atividades de computação desplugada na Educação Básica. In: Anais do Workshop de Informática na Escola (Vol. 21, No. 1, p. 256).

Gomes, T.; Melo, J. e Tedesco, P. (2016). Jogos Digitais no Ensino de Conceitos de Programação para Crianças. In: Brazilian Symposium on Computers in Education (Simpósio Brasileiro de Informática na Educação-SBIE) (Vol. 27, No. 1, p. 470).

Raabe, A.; Zanchett, G. e Vahldick, A. (2015). Jogos de Programar como uma Abordagem para os Primeiros Contatos dos Estudantes com à Programação. In: Anais dos Workshops do Congresso Brasileiro de Informática na Educação (Vol. 4, No. 1, p. 1485).

Silva, T. R.; Medeiros, T. J. e Aranha, E. H. D. S. (2014). Jogos digitais para ensino e aprendizagem de programação: uma revisão sistemática da literatura. In: Brazilian Symposium on Computers in Education (Simpósio Brasileiro de Informática na Educação-SBIE) (Vol. 25, No. 1, p. 692).

Vieira, A.; Passos, O. e Barreto, R. (2013). Um relato de experiência do uso da técnica computação desplugada. Anais do XXI WEI, 670-679.

Wing, J. M. (2006). Computational thinking. Communications of the ACM, 49(3), 3335.

Zanetti, H.; Borges, M. e Ricarte, I. (2016). Pensamento Computacional no Ensino de Programação: Uma Revisão Sistemática da Literatura Brasileira. In: Brazilian Symposium on Computers in Education (Simpósio Brasileiro de Informática na Educação-SBIE) (Vol. 27, No. 1, p. 21). 